\title{
Cortical Thickness in Alcohol Dependent Patients With Apathy
}

\author{
Kebing Yang ${ }^{1}$, Qingyan Yang ${ }^{1}$, Yajuan Niu ${ }^{1}$, Fengmei Fan ${ }^{1}$, Song Chen ${ }^{1}$, Xingguang Luo ${ }^{2}$, \\ Shuping Tan ${ }^{1}$, Zhiren Wang ${ }^{1}$, Jinghui Tong ${ }^{1}$, Fude Yang ${ }^{1}$, Thang M. Le ${ }^{3}$, \\ Chiang-Shan R. Li ${ }^{2,3}$ and Yunlong Tan ${ }^{1 *}$ \\ 1 Peking University Huilongguan Clinical Medical School, Beijing Huilongguan Hospital, Beijing, China, ${ }^{2}$ Department of \\ Psychiatry, Yale University School of Medicine, New Haven, CT, United States, ${ }^{3}$ Department of Neuroscience, Yale \\ University School of Medicine, New Haven, CT, United States
}

OPEN ACCESS

Edited by:

Yanhui Liao,

Sir Run Run Shaw Hospital,

China

Reviewed by: Wei Hao,

Central South University,

China

Shankar Tumati,

Univeristy of Ottawa, Canada

*Correspondence:

Yunlong Tan

yltan21@126.com

Specialty section:

This article was submitted to

Addictive Disorders,

a section of the journal

Frontiers in Psychiatry

Received: 25 February 2020

Accepted: 14 April 2020

Published: 05 May 2020

Citation:

Yang K, Yang Q, Niu Y, Fan F, Chen S, Luo X, Tan S, Wang Z, Tong J, Yang F.

Le TM, Li C-SR and Tan Y (2020)

Cortical Thickness in Alcohol

Dependent Patients With Apathy.

Front. Psychiatry 11:364.

doi: 10.3389/fpsyt.2020.00364
Objectives: Many studies reported structural brain changes in patients with alcohol dependence (PADs). However, no studies identified structural correlates of apathy that might aggravate alcohol misuse. Here, we explored regional differences in cortical thickness in PADs relative to healthy controls ( $\mathrm{HCs}$ ), and examined the potential correlation of regional thickness with the severity of apathy.

Methods: Magnetic resonance imaging data were collected from 33 male PADs and 35 male age- and education-matched HCs. We used the FreeSurfer software to investigate group differences in cortical thickness across 148 regions. Apathy was evaluated using the Lille Apathy Rating Scale-Informant (LARS-I). Regression analyses examined the relationship between cortical thickness of regions of interest and apathy score in PADs.

Results: Compared to HCs, PADs showed significant decreases in the cortical thickness of occipito-temporal cortex (OTC), including the left middle occipital gyrus and occipital pole, right superior occipital gyri, and bilateral lingual gyrus; bilateral superior parietal cortex (SPC), including the right intraparietal sulcus; and bilateral inferior parietal cortex (IPC). Furthermore, the cortical thickness of all of the three regions was negatively correlated with the apathy total scores. The cortical thickness of the IPC was also negatively correlated with the action initiation subscore of the LARS-I.

Conclusions: The current results suggest the thickness of bilateral parietal and occipital temporal cortices as neural markers of apathy in PADs. These findings add to the literature by identifying the neural bases of a critical clinical feature of individuals with alcoholism.

Keywords: alcohol dependence, apathy, occipito-temporal cortex, superior parietal cortex, inferior parietal cortex

\section{INTRODUCTION}

Alcohol dependence $(\mathrm{AD})$ is a chronic illness characterized by poor treatment outcomes and high relapse rates (1). Individuals with $\mathrm{AD}$ frequently demonstrate motivational deficits, exacerbating personal and interpersonal health (2). Brain imaging was widely used to investigate the consequences of alcohol misuse, which included atrophy of the gray and white matters, sulcal widening, and ventricular enlargement. The structural changes resulted from excessive alcohol 
consumption (3-7). These deficits involved multiple brain networks that support executive control, salience response, and emotion regulation, which may directly or indirectly contribute to cognitive and motivational dysfunction in patients with $\mathrm{AD}$ $(8,9)$.

The main clinical feature of apathy is lack of motivation (10) and reduced initiative, interest, and/or concern (11). It is also proposed that apathy be defined as dysfunction of volitional processes or a reduction in self-generated, voluntary, and purposeful behavior (i.e., goal-directed behavior) (12). Difficulties in goal-directed behavior, as a key feature of apathy, was significantly associated with symptoms of alcohol dependence (13). Almost all patients with severe alcohol dependence show diminished or lack of living practices except for drinking-related events, and executive dysfunction on alcohol abstinence (2, 14, 15). Importantly, patients undergoing treatment for $\mathrm{AD}$ often relapse despite better knowledge and their intention to remain abstinent, reflecting impairments in volition and goal-directed behavior (16). It is thus critical to investigate the neural correlates of apathy in $\mathrm{AD}$.

In imaging studies of various brain disorders, apathy has been associated with structural and function deficits of multiple brain regions (17), particularly the dorsal anterior cingulate cortex (dACC) (18). Other studies implicated the fronto-subcortical circuits and lateral parietal cortex (19). Numerous studies have also shown structural and functional deficits of a wide range of cortical and subcortical circuits in individuals with AD (20). However, no studies to our knowledge have specifically examined the cerebral structural bases of apathy in AD.

The current study addressed this gap in research. On the basis of the literature that goal-directed behaviors require top-down attentional, cognitive and affective control as well as execution of complex motor movements (21), we hypothesized that the frontal and parietal cortical thickness would be diminished and may represent a neurobiological marker of apathy in AD.

\section{METHODS}

\section{Participants and Consents}

All patients with $\mathrm{AD}$ (PADs) were males, of Han Chinese descent, and recruited from Beijing Huilongguan Hospital between 2017 and 2018. Inclusion criteria were as follows: a) age between 18 and 65 years; b) diagnosis of $\mathrm{AD}$ in accordance with Diagnostic and Statistical Manual of Mental Disorders (DSM-IV-TR) criteria (22); c) right-handed; d) abstinent for 14 to 28 days after completion of medically assisted withdrawal, with no obvious withdrawal symptoms and a Clinical Institute Withdrawal Assessment for Alcohol (CIWA) (23) score of less than 3. Patients with other axis I mental disorders, including dependence on substances other than alcohol and nicotine, or severe medical conditions (e.g., unstable hypertension, poorly controlled diabetes, myocardial infarction, liver cirrhosis) were excluded. In addition, we recruited 35 male age- and educationmatched healthy controls (HCs) from the general medical clinics of the hospital and local community. Prior to study inclusion, all participants provided written informed consent in accordance with the Declaration of Helsinki and a protocol approved by the ethics committee of Beijing Huilongguan Hospital.

\section{Assessments}

All PADs were evaluated with the Alcohol Use Disorder Identification Test (AUDIT) (24), a semi-structured questionnaire to investigate the severity of alcohol consumption (25). Lifetime Drinking History assessment to determine the number of daily drinks (one drink $=10 \mathrm{~g}$ of ethanol), age at first drink, and age at onset of alcohol dependence. The extent of apathy was assessed using the Lille Apathy Rating Scale-Informant (LARS-I) (11), which comprised 33 items across nine domains, each corresponding to a specific clinical dimension of apathy. Each sub-scale score was from -4 to +4 , with higher positive scores indicating more severe symptoms. In brief, a negative or positive score indicates less or more apathy, and zero represents moderate apathy in everyday productivity and interests domains or non-applicable or non-classifiable reply in other domains. In this study, we focused on the apathy total score (T score) and action initiation (AI) subscore in data analyses.

\section{MRI Data Acquisition and Analyses}

All PADs (after detoxification) and HCs underwent MRI. MRIs were obtained by using a Siemens 3T MRI scanner. Head motion was minimized using foam pads. Structural MR images were acquired for the whole brain with a sagittal 3D-magnetization prepared rapid acquisition gradient echo (MPRAGE) sequence, using the follow parameters: echo time $(\mathrm{TE})=2.98 \mathrm{~ms}$, inversion time $(\mathrm{TI})=900 \mathrm{~ms}$, repetition time $(\mathrm{TR})=2,300 \mathrm{~ms}$, flip angle $(\mathrm{FA})=9^{\circ}$, field-of-view $(\mathrm{FOV})=240 \mathrm{~mm} \times 256 \mathrm{~mm}$, matrix size $=$ $256 \times 240$, thickness $/$ gap $=1 / 0 \mathrm{~mm}$.

Measures of cortical thickness (mm) of 148 cortical regions (bilateral; $74 \times 2$; Desikan-Killiany Atlas), total subcortical thickness, and total gray matter volume (TGV) were obtained using FreeSurfer (26) (http://surfer.nmr.mgh.harvard.edu). TGV was used as a covariate in all analyses to account for differences in head size. We followed the ENIGMA pipeline (http://enigma. ini.usc.edu/) for quality control of the images: All cortical regions with a thickness $>1.5$ or $<1.5$ times the interquartile range were identified and visually inspected by overlaying their segmentations on the participants' anatomical images. Only imaging data for which segmentation was judged to be accurate were subjected to statistical analyses.

\section{Statistical Analysis}

As all participants were male, continuous demographic variables were analyzed using independent samples t-tests and categorical data were analyzed with Chi-square $\left(\chi^{2}\right)$ tests to compare PADs and HCs. The level of statistical significance was set at $p<0.05$ (two-tailed).

Cortical thickness values of the 148 regions were compared between PADs and HCs using univariate linear regression analysis, where the cortical thickness of each region was used as the dependent variable, and group (PADs/HCs), age, smoking status, education, and TGV were entered as independent 
variables. The threshold for statistical significance was set at $p<$ 0.00033784 (i.e., $0.05 / 148$ ) to correct for multiple comparisons (Bonferroni correction).

We identified 12 cortical regions of interest (ROIs) that differed significantly between PADs and HCs in thickness, and these regions belong to the occipital temporal cortex (OTC) (lateral cortex including the left middle occipital gyrus, bilateral lingual gyrus, left occipital pole and right superior occipital gyrus), superior parietal cortex (SPC) (lateral cortex including the right intraparietal sulcus and bilateral superior parietal lobule), and inferior parietal cortex (IPC) (lateral cortex including the bilateral angular gyrus and supramarginal gyrus) (27). We performed bivariate correlation analyses to explore the relationship between cortical thickness of the OTC, SPC and IPC and apathy total score (T score), and action initiation subscore (AI score) in PADs. Statistical significance was tested at $p<0.05 / 6$, two-sided, to correct for multiple comparisons. As many alcohol drinkers are heavy smokers, we performed two sets of regression, one without smoking status as a covariate, so that the results best reflect the typical populations of alcoholic participants, and the other with smoking status as a covariate, so the effects of smoking could be controlled for.

\section{RESULTS}

\section{Demographic and Clinical Characteristics}

There were no significant differences in age $(t=1.257, p=0.213)$ or years of education $(t=-0.883, p=0.381)$ between PADs and HCs. More PADs were smokers than HCs $\left(\chi^{2}=11.87, p=0.001\right)$. Smoking status was thus included as a covariate in the following data analyses. Compared to HCs, PADs showed higher LARS total or T score $(t=5.85, p<0.001)$ and action initiation or AI subscore $(t=5.43, p<0.001)$. The demographic and clinical characteristics of the two groups are presented in Table 1.

\section{Cortical Thickness}

The cortical thickness of the bilateral lingual gyrus in the OTC was significantly higher in PADs as compared with $\mathrm{HCs}$; however, relative to HCs, PADs exhibited significant decreases

TABLE 1 | Demographic and clinical characteristics in patients with alcohol dependence (PADs) and healthy controls (HCs) (mean $\pm \mathrm{SD}$ ).

\begin{tabular}{lcccc}
\hline & PAD (n=33) & HCs $(\mathbf{n}=\mathbf{3 5})$ & $\boldsymbol{t} / \chi^{2}$-value & $\boldsymbol{p}$-value \\
\hline Age, years & $44.8 \pm 9.8$ & $41.6 \pm 11.5$ & 1.257 & 0.213 \\
Education, years & $11.8 \pm 3.3$ & $12.3 \pm 2.4$ & -0.883 & 0.381 \\
Smoker/non-smoker $^{\mathrm{a}}$ & $26 / 7$ & $13 / 22$ & 11.87 & 0.001 \\
Action initiation (Al) & $-1.21 \pm 0.86$ & $-2.19 \pm 0.61$ & 5.43 & 0.000 \\
Total score of LARS & $-5.85 \pm 6.87$ & $-14.78 \pm 5.70$ & 5.85 & 0.000 \\
Age at onset of AD, years & $32.5 \pm 10.1$ & $\mathrm{~N} / \mathrm{A}$ & $\mathrm{N} / \mathrm{A}$ & $\mathrm{N} / \mathrm{A}$ \\
Age at first drink, years & $19.9 \pm 5.5$ & $\mathrm{~N} / \mathrm{A}$ & $\mathrm{N} / \mathrm{A}$ & $\mathrm{N} / \mathrm{A}$ \\
AUDIT total score & $21.7 \pm 5.2$ & $\mathrm{~N} / \mathrm{A}$ & $\mathrm{N} / \mathrm{A}$ & $\mathrm{N} / \mathrm{A}$ \\
Mean daily drinks ${ }^{\mathrm{b}}$ & $19.1 \pm 8.7$ & $\mathrm{~N} / \mathrm{A}$ & $\mathrm{N} / \mathrm{A}$ & $\mathrm{N} / \mathrm{A}$
\end{tabular}

PADs, patients with alcohol dependence; HCs, healthy controls; LARS, Lille Apathy Rating Scale; AUDIT, Alcohol Use Disorders Identification Test; N/A, not applicable.

${ }^{a}$ Chi-square $\left(\chi^{2}\right)$ test: smoker = current or previous smoker; non-smoker: never smoker

${ }^{b}$ One drink contains $10 \mathrm{~g}$ of pure alcohol. in overall cortical thickness in the OTC (consisting of the left middle occipital gyrus and occipital pole, right superior occipital gyri, and bilateral lingual gyrus), SPC, as well as IPC, (all p's $<0.00033784$, Table 2) accounting for age, years of education, smoking status, and TGV. The mean cortical thickness and statistics for all 148 regions were shown in Supplementary Table 1.

For PADs, we focused on the OTC, SPC, and IPC and performed Pearson correlation analyses to evaluate the relationship between cortical thickness and apathy ( $\mathrm{T}$ score and AI subscore). Evaluated at $p<0.0083$ (i.e., 0.05/6, Bonferroni correction), the results revealed negative correlations between the thickness of the OTC, SPC as well as IPC and both apathy T score. The cortical thickness of the IPC was also negatively correlated with the AI subscore. These results are shown in Figure 1.

Because many more PADs are smokers, we performed covariance analyses with smoking status as a covariate. The results showed that apathy $\mathrm{T}$ score remained negatively correlated with the thickness of the OTC $(r=-0.44, p=0.011)$, IPC ( $r=-0.47, p=0.007)$, and SPC $(r=-0.58, p<0.001)$; and the apathy AI subscore showed negative correlation with thickness of the IPC $(r=-0.41, p=0.019)$ and SPC $(r=-0.44, p=0.012)$ but not the OTC ( $r=-0.32, p=0.078)$. The findings suggest that after controlling for smoking status, the thickness of the parietal, but not that of occipital temporal cortex, was correlated with difficulties in action initiation in PADs.

\section{DISCUSSION}

We hypothesized that PADs would exhibit reduced thickness in the frontal and parietal cortex, and the extent of the reduction would be correlated with the severity of apathy in PADs. To this end, we determined differences in regional cortical thickness

TABLE 2 | Brain regions with significant differences in cortical thickness between patients with alcohol dependence (PADs) and healthy controls (HCs).

\begin{tabular}{|c|c|c|c|c|}
\hline $\begin{array}{l}\text { Cortical thickness of } \\
\text { Destrieux Atlas (mm) }\end{array}$ & PADs (n 33) & HCs $(n=35)$ & $\begin{array}{c}F- \\
\text { value }\end{array}$ & $p$-value \\
\hline L-Middle occipital gyrus & $2.165 \pm 0.297$ & $2.613 \pm 0.123$ & 29.545 & 9.768E-07 \\
\hline L-Lingual gyrus & $2.118 \pm 0.142$ & $1.879 \pm 0.131$ & 44.499 & 7.960E-09 \\
\hline L-Angular gyrus & $2.152 \pm 0.329$ & $2.705 \pm 0.139$ & 26.065 & 3.378E-06 \\
\hline L-Supramarginal gyrus & $2.371 \pm 0.271$ & $2.741 \pm 0.141$ & 14.574 & 3.138E-04 \\
\hline $\begin{array}{l}\text { L-Superior parietal } \\
\text { lobule }\end{array}$ & $1.931 \pm 0.301$ & $2.461 \pm 0.117$ & 32.666 & 3.351E-07 \\
\hline L-Occipital pole & $1.672 \pm 0.219$ & $1.947 \pm 0.145$ & 20.949 & 2.321E-05 \\
\hline $\begin{array}{l}\text { R-Superior occipital } \\
\text { gyrus }\end{array}$ & $1.881 \pm 0.302$ & $2.261 \pm 0.145$ & 17.749 & 8.313E-05 \\
\hline R-Lingual gyrus & $2.137 \pm 0.129$ & $1.967 \pm 0.156$ & 16.656 & 1.303E-04 \\
\hline R-Angular gyrus & $2.167 \pm 0.324$ & $2.704 \pm 0.131$ & 41.123 & 2.208E-08 \\
\hline R-Supramarginal gyrus & $2.389 \pm 0.299$ & $2.741 \pm 0.118$ & 16.734 & 1.262E-04 \\
\hline $\begin{array}{l}\text { R-Superior parietal } \\
\text { lobule }\end{array}$ & $1.929 \pm 0.318$ & $2.445 \pm 0.118$ & 38.473 & $5.044 \mathrm{E}-08$ \\
\hline $\begin{array}{l}\text { R-Intraparietal sulcus } \\
\text { and transverse parietal } \\
\text { sulci }\end{array}$ & $1.892 \pm 0.211$ & $2.191 \pm .089$ & 20.290 & 3.004E-05 \\
\hline
\end{tabular}

One-way ANOVA, Bonferroni corrected at $p<0.00033784$. 

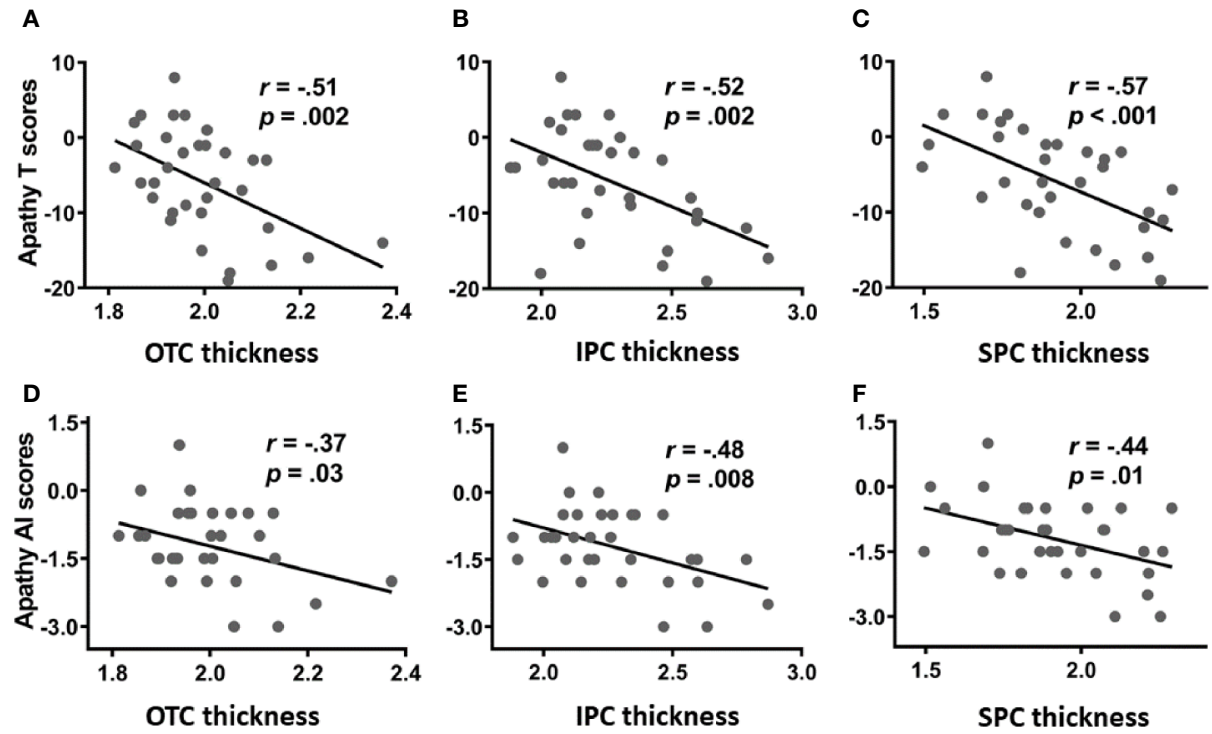

FIGURE 1 | Correlation of cortical thickness of the occipital temporal cortex (OTC), inferior parietal cortex (IPC), and superior parietal cortex (SPC) with apathy total score (T score; upper panel: A-C) and action initiation subscore (Al subscore; lower panel: D-F).

between PADs and HCs and investigated how these differences are related to apathy in PADs. The results suggest that $\mathrm{AD}$ was associated with reduced thickness in the bilateral occipital temporal cortex, the superior and inferior parietal cortices, and the extent of the reduction in cortical thickness was correlated with the severity of apathy. Although the cortical thickness of the bilateral lingual gyrus showed peculiar increases in PADs, this change, contrary to that shown in most of the previous studies (5, 28), did not affect the reduction of the whole OTC region in PADs compared with HCs. The mechanism of compensatory hypertrophy might be a potential explanation to this change (29) because a previous study found similar increases in alcohol abuse individuals, regardless of sex and brain regions (30). Greater reduction of parietal cortical thickness appears to be more specifically related to action initiation deficits in PADs. Together, these findings support structural changes of posterior cortical regions as a neural marker of apathy in alcoholism.

Structural imaging studies reported significant volume losses in the prefrontal cortex, insula, and hippocampus in $\operatorname{AD}(5,31,32)$; however, volumetric deficits of the posterior cortical regions have received less attention (30). For instance, parietal-occipital gray matter volumes were decreased in patients with $\mathrm{AD}$ and can be used to predict shorter time of any alcohol use and relapse to heavy drinking (33). A recent meta-analysis of 3,240 individuals with drug and alcohol dependence indicated that the most severe volumetric deficits are found in not only the frontal but also occipital, temporal, and parietal cortices in those with alcohol dependence (34). Consistently, we observed a significant reduction in cortical thickness in the occipital and parietal cortices. However, it is unclear why structural changes cannot be detected in the frontal cortex. One possibility is that the current work only focused on cortical thickness, a volumetric measure different from that as employed in the earlier work. This discrepancy clearly warrants further studies.

Many studies examined the neural mechanisms underlying cognitive and emotional deficits (35) but few focused on apathy in individuals with $\mathrm{AD}$ (36). In accordance with previous studies we observed more significant apathy symptoms, as reflected in the apathy total score in PADs than in HCs, suggesting motivational dysfunction in individuals with alcoholism (37). The cortical thickness of the occipital-temporal as well as superior and inferior parietal cortices was negatively correlated with the severity of apathy, consistent with earlier work associating apathy with structural changes in the parietal cortex, in addition to the prefrontal cortex (PFC) and basal ganglia (38). Research has indicated that the superior parietal lobule, including the intraparietal sulcus, play a key role in goaldirected behaviors and volitional processes (39). In addition, an [18F]-fluorodeoxyglucose (FDG) positron emission tomography (PET) study in a rat model of alcoholism revealed that alcohol significantly reduced whole-brain glucose metabolism, and that the effect was most pronounced in the parietal cortex (40). Together, the current along with these earlier studies support the importance of posterior cortical structures in mediating cognitive and emotional deficits (41), which may manifest as apathy in $\mathrm{AD}$. Importantly, as apathy may impact the motivation for behavioral changes, the current findings are also consistent with our earlier work showing that volumetric deficits in the posterior-occipital region predicted relapse in $\mathrm{AD}$ patients undergoing treatment (42). 


\section{Limitations}

A few limitations of present study included: 1) there were more smokers in PADs than in HCs. Although we have accounted for the potential effects of smoking in data analyses, one cannot completely rule out the effects of chronic smoking on the findings. On the other hand, compared to smoking, alcohol consumption appears to be an overriding factor leading to alterations in brain structures and functions (43). Further, alcohol dependent individuals are typically smokers; thus, one may argue that the current findings reflect that general alcohol dependent populations; 2) as a specialist hospital in Beijing for the PADs, we were exposed to patients with more serious clinical symptoms and social dysfunction and difficult to enroll those patients who have mild symptoms and no obvious apathy. This is the reason of correlation analysis without setting apathy-free patients as control in this study. 3) because there were much fewer women with alcohol dependence in China and no women were included in the present study, we were unable to examine the effects of gender on cortical thickness in relation to the impact of alcohol misuse. As sex differences in the etiological processes or consequences of alcohol misuse have been demonstrated in numerous studies $(44,45)$. We hope to study more women to examine sex differences, including the finding that neurotoxicity may be more severe among women with alcoholism. Lastly, the sample size of the present study is moderate and the findings would warrant replication.

\section{CONCLUSIONS}

The present study demonstrated significant differences in occipital, temporal, and parietal cortical thickness in individual with alcohol dependence as compared with healthy controls. These structural changes were related to the severity of apathy in alcohol dependent individuals. Future research with functional imaging may provide an opportunity to understand in more details how the posterior cortical regions participate in motivated behavior and how these processes may be compromised in alcohol dependent individuals with apathy.

\section{DATA AVAILABILITY STATEMENT}

All datasets generated for this study are included in the article/ Supplementary Material.

\section{REFERENCES}

1. World Health Organization. Global status report on alcohol and health. Geneva, Switzerland: World Health Organization (2011). 286 p.

2. Day AM, Kahler CW, Ahern DC, Clark US. Executive Functioning in Alcohol Use Studies: A Brief Review of Findings and Challenges in Assessment. Curr Drug Abuse Rev (2015) 8(1):26-40. doi: 10.2174/187447370866615041 6110515

\section{ETHICS STATEMENT}

The studies involving human participants were reviewed and approved by the Ethics Committee of Beijing Huilongguan Hospital. The patients/participants provided their written informed consent to participate in this study. Written informed consent was obtained from the individual(s) for the publication of any potentially identifiable images or data included in this article.

\section{AUTHOR CONTRIBUTIONS}

YT designed the project, and took responsibility for the integrity of the data and the accuracy of the data analysis. KY, QY, and YN were responsible for recruiting the patients, performing the clinical rating, and collecting the samples. KY wrote the paper. Critical revision of the manuscript for important intellectual content was drafted by YT and C-SL. C-SL, XL, ST, ZW, JT, SC, FF, FY, and TL were invited in evolving the ideas, statistical analysis, and editing the manuscript. All authors have contributed to and have approved the final manuscript.

\section{FUNDING}

This work was supported by the Beijing Municipal Administration of Hospitals Clinical medicine Development of special funding support (XMLX201834), Beijing Municipal Commission of Science and Technology (Z161100000516046), and the National Institutes of Health (AA021449). None of the funding sources influenced the study design, the collection, analysis, and interpretation of the data, approval of the manuscript, or the decision to submit the manuscript for publication.

\section{ACKNOWLEDGMENTS}

We would like to acknowledge all participants involved in this study.

\section{SUPPLEMENTARY MATERIAL}

The Supplementary Material for this article can be found online at: https://www.frontiersin.org/articles/10.3389/fpsyt.2020. 00364/full\#supplementary-material

3. Dupuy M, Chanraud S. Imaging the Addicted Brain: Alcohol. Int Rev Neurobiol (2016) 129:1-31. doi: 10.1016/bs.irn.2016.04.003

4. Monnig MA, Tonigan JS, Yeo RA, Thoma RJ, McCrady BS. White matter volume in alcohol use disorders: a meta-analysis. Addict Biol (2013) 18 (3):581-92. doi: 10.1111/j.1369-1600.2012.00441.x

5. Sutherland GT, Sheedy D, Kril JJ. Neuropathology of alcoholism. Handb Clin Neurol (2014) 125:603-15. doi: 10.1016/B978-0-444-62619-6.00035-5 
6. Topiwala A, Ebmeier KP. Effects of drinking on late-life brain and cognition. Evid Based Ment Health (2018) 21(1):12-5. doi: 10.1136/eb-2017-102820

7. Xiao P, Dai Z, Zhong J, Zhu Y, Shi H, Pan P. Regional gray matter deficits in alcohol dependence: A meta-analysis of voxel-based morphometry studies. Drug Alcohol Depend (2015) 153:22-8. doi: 10.1016/j.drugalcdep.2015.05.030

8. Koob GF, Buck CL, Cohen A, Edwards S, Park PE, Schlosburg JE, et al. Addiction as a stress surfeit disorder. Neuropharmacology (2014) 76 Pt B:37082. doi: 10.1016/j.neuropharm.2013.05.024

9. Koob GF, Volkow ND. Neurobiology of addiction: a neurocircuitry analysis. Lancet Psychiat (2016) 3(8):760-73. doi: 10.1016/S2215-0366(16)00104-8

10. Starkstein SE, Leentjens AF. The nosological position of apathy in clinical practice. J Neurol Neurosurg Psychiatry (2008) 79(10):1088-92. doi: 10.1136/ jnnp.2007.136895

11. Sockeel P, Dujardin K, Devos D, Deneve C, Destee A, Defebvre L. The Lille apathy rating scale (LARS), a new instrument for detecting and quantifying apathy: validation in Parkinson's disease. J Neurol Neurosurg Psychiatry (2006) 77(5):579-84. doi: 10.1136/jnnp.2005.075929

12. Levy R, Dubois B. Apathy and the functional anatomy of the prefrontal cortex-basal ganglia circuits. Cereb Cortex (2006) 16(7):916-28. doi: 10.1093/ cercor/bhj043

13. Paulus DJ, Bakhshaie J, Lemaire C, Garza M, Ochoa-Perez M, Valdivieso J, et al. Negative Affectivity and Problematic Alcohol Use Among Latinos in Primary Care: The Role of Emotion Dysregulation. J Dual Diagn (2016) 12 (2):137-47. doi: 10.1080/15504263.2016.1172897

14. Sher KJ. The Oxford handbook of substance use and substance use disorders. Oxford University Press (2016). 2 p.

15. Bosscher RJ, Smit JH. Confirmatory factor analysis of the General Self-Efficacy Scale. Behav Res Ther (1998) 36(3):339-43. doi: 10.1016/s0005-7967(98)00025-4

16. Garbusow M, Sebold M, Beck A, Heinz A. Too difficult to stop: mechanisms facilitating relapse in alcohol dependence. Neuropsychobiology (2014) 70 (2):103-10. doi: 10.1159/000362838

17. Kos C, van Tol MJ, Marsman JB, Knegtering H, Aleman A. Neural correlates of apathy in patients with neurodegenerative disorders, acquired brain injury, and psychiatric disorders. Neurosci Biobehav Rev (2016) 69:381-401. doi: 10.1016/j.neubiorev.2016.08.012

18. McIntosh RC, Rosselli M, Uddin LQ, Antoni M. Neuropathological sequelae of Human Immunodeficiency Virus and apathy: A review of neuropsychological and neuroimaging studies. Neurosci Biobehav Rev (2015) 55:147-64. doi: 10.1016/j.neubiorev.2015.04.008

19. Stella F, Radanovic M, Aprahamian I, Canineu PR, de Andrade LP, Forlenza OV. Neurobiological correlates of apathy in Alzheimer's disease and mild cognitive impairment: a critical review. J Alzheimers Dis (2014) 39(3):633-48. doi: 10.3233/JAD-131385

20. Shirer WR, Ryali S, Rykhlevskaia E, Menon V, Greicius MD. Decoding subject-driven cognitive states with whole-brain connectivity patterns. Cereb Cortex (2012) 22(1):158-65. doi: 10.1093/cercor/bhr099

21. Gallivan JP, McLean DA, Valyear KF, Pettypiece CE, Culham JC. Decoding action intentions from preparatory brain activity in human parieto-frontal networks. J Neurosci (2011) 31(26):9599-610. doi: 10.1523/JNEUROSCI.0080-11.2011

22. American Psychiatric Association. Electronic DSM-IV-TR plus. Version 1.0. ed. Washington, D.C.: American Psychiatric Association (2000), 1 p.

23. Sullivan JT, Sykora K, Schneiderman J, Naranjo CA, Sellers EM. Assessment of alcohol withdrawal: the revised clinical institute withdrawal assessment for alcohol scale (CIWA-Ar). Br J Addict (1989) 84(11):1353-7. doi: 10.1111/ j.1360-0443.1989.tb00737.x

24. Saunders JB, Aasland OG, Babor TF, de la Fuente JR, Grant M. Development of the Alcohol Use Disorders Identification Test (AUDIT): WHO Collaborative Project on Early Detection of Persons with Harmful Alcohol Consumption-II. Addiction (1993) 88(6):791-804. doi: 10.1111/j.1360-0443.1993.tb02093.x

25. Sobell LC, Brown J, Leo GI, Sobell MB. The reliability of the Alcohol Timeline Followback when administered by telephone and by computer. Drug Alcohol Depend (1996) 42(1):49-54. doi: 10.1016/0376-8716(96)01263-X
26. Fischl B. FreeSurfer. Neuroimage (2012) 62(2):774-81. doi: 10.1016/ j.neuroimage.2012.01.021

27. Destrieux C, Fischl B, Dale A, Halgren E. Automatic parcellation of human cortical gyri and sulci using standard anatomical nomenclature. Neuroimage (2010) 53(1):1-15. doi: 10.1016/j.neuroimage.2010.06.010

28. Buhler M, Mann K. Alcohol and the human brain: a systematic review of different neuroimaging methods. Alcohol Clin Exp Res (2011) 35(10):1771-93. doi: 10.1111/j.1530-0277.2011.01540.x

29. Bansal R, Hellerstein DJ, Peterson BS. Evidence for neuroplastic compensation in the cerebral cortex of persons with depressive illness. Mol Psychiatry (2018) 23(2):375-83. doi: 10.1038/mp.2017.34

30. Fein G, Greenstein D, Cardenas VA, Cuzen NL, Fouche JP, Ferrett H, et al. Cortical and subcortical volumes in adolescents with alcohol dependence but without substance or psychiatric comorbidities. Psychiatry Res (2013) 214(1):1-8. doi: 10.1016/j.pscychresns.2013. 06.001

31. van Eijk J, Demirakca T, Frischknecht U, Hermann D, Mann K, Ende G. Rapid partial regeneration of brain volume during the first 14 days of abstinence from alcohol. Alcohol Clin Exp Res (2013) 37(1):67-74. doi: 10.1111/j.1530-0277.2012.01853.x

32. Hoefer ME, Pennington DL, Durazzo TC, Mon A, Abe C, Truran D, et al. Genetic and behavioral determinants of hippocampal volume recovery during abstinence from alcohol. Alcohol (2014) 48(7):631-8. doi: 10.1016/ j.alcohol.2014.08.007

33. Rando K, Hong KI, Bhagwagar Z, Li CS, Bergquist K, Guarnaccia J, et al. Association of frontal and posterior cortical gray matter volume with time to alcohol relapse: a prospective study. Am J Psychiatry (2011) 168(2):183-92. doi: 10.1176/appi.ajp.2010.10020233

34. Mackey S, Allgaier N, Chaarani B, Spechler P, Orr C, Bunn J, et al. MegaAnalysis of Gray Matter Volume in Substance Dependence: General and Substance-Specific Regional Effects. Am J Psychiatry (2019) 176(2):119-28. doi: 10.1176/appi.ajp.2010.10020233

35. Heilig M, Egli M, Crabbe JC, Becker HC. Acute withdrawal, protracted abstinence and negative affect in alcoholism: are they linked? Addict Biol (2010) 15(2):169-84. doi: 10.1111/j.1369-1600.2009.00194.x

36. Tumati S, Martens S, de Jong BM, Aleman A. Lateral parietal cortex in the generation of behavior: Implications for apathy. Prog Neurobiol (2019) 175:20-34. doi: 10.1016/j.pneurobio.2018.12.003

37. Clark US, Oscar-Berman M, Shagrin B, Pencina M. Alcoholism and judgments of affective stimuli. Neuropsychology (2007) 21(3):346-62. doi: 10.1037/0894-4105.21.3.346

38. Lundqvist T. Imaging cognitive deficits in drug abuse. Curr Top Behav Neurosci (2010) 3:247-75. doi: 10.1007/7854_2009_26

39. Andersen RA, Cui H. Intention, action planning, and decision making in parietal-frontal circuits. Neuron (2009) 63(5):568-83. doi: 10.1016/ j.neuron.2009.08.028

40. Gispert JD, Figueiras FP, Vengeliene V, Herance JR, Rojas S, Spanagel R. Changes in cerebral [(18)F]-FDG uptake induced by acute alcohol administration in a rat model of alcoholism. Behav Brain Res (2017) 327:29-33. doi: 10.1016/j.bbr.2017.03.038

41. Siever LJ. Neurobiology of aggression and violence. Am J Psychiatry (2008) 165(4):429-42. doi: 10.1176/appi.ajp.2008.07111774

42. Wang J, Fan Y, Dong Y, Ma M, Dong Y, Niu Y, et al. Combining gray matter volume in the cuneus and the cuneus-prefrontal connectivity may predict early relapse in abstinent alcohol-dependent patients. PloS One (2018) 13(5): e196860. doi: 10.1371/journal.pone.0196860

43. Ide JS, Zhornitsky S, Hu S, Zhang S, Krystal JH, Li CR. Sex differences in the interacting roles of impulsivity and positive alcohol expectancy in problem drinking: A structural brain imaging study. NeuroImage: Clin (2017) 14:7509. doi: 10.1016/j.nicl.2017.03.015

44. Ide JS, Zhornitsky S, Chao HH, Zhang S, Hu S, Wang W, et al. Thalamic Cortical Error-Related Responses in Adult Social Drinkers: Sex Differences and Problem Alcohol Use. Biol Psychiatry Cogn Neurosci Neuroimaging (2018) 3(10):868-77. doi: 10.1016/j.bpsc.2018.04.008 
45. Filbey FM, Aslan S, Calhoun VD, Spence JS, Damaraju E, Caprihan A, et al. Long-term effects of marijuana use on the brain. Proc Natl Acad Sci U S A (2014) 111(47):16913-8. doi: 10.1073/pnas.1415297111

Conflict of Interest: The authors declare that the research was conducted in the absence of any commercial or financial relationships that could be construed as a potential conflict of interest.
Copyright (c) 2020 Yang, Yang, Niu, Fan, Chen, Luo, Tan, Wang, Tong, Yang, Le, Li and Tan. This is an open-access article distributed under the terms of the Creative Commons Attribution License (CC BY). The use, distribution or reproduction in other forums is permitted, provided the original author(s) and the copyright owner(s) are credited and that the original publication in this journal is cited, in accordance with accepted academic practice. No use, distribution or reproduction is permitted which does not comply with these terms. 